\title{
Proposição de um Algoritmo para Classificação de Desempenho Municipal em Sustentabilidade
}

\author{
Marcelo Santana Camacho' ${ }^{1,2}$, Fabrício de Souza Farias ${ }^{1,3}$ \\ 1Programa de Pós-Graduação em Computação Aplicada \\ Universidade Federal do Pará (UFPA) - Tucuruí, PA - Brasil \\ ${ }^{2}$ Campus Universitário de Salinópolis - Universidade Federal do Pará (UFPA), Brasil \\ ${ }^{3}$ Campus Universitário de Cametá - Universidade Federal do Pará (UFPA), Brasil \\ \{camacho, fabriciosf\}@ufpa.br
}

\begin{abstract}
The Sustainability Barometer has stood out as one of the main methodologies for creating sustainability indicators. In this work we present a proposal called 'barometroR', based on an algorithm that aims to simplify the number of indications for the origin of the sustainability index. To validate the results, two pre-processing methods for feature selection are compared with the original model based on the literature, in a case study for 13 municipalities, in which 26 factors are evaluated. The results demonstrate that the proposed methodology guarantees the same Barometer score with a $95 \%$ confidence interval defined by 10 attributes.
\end{abstract}

Resumo. O Barômetro da Sustentabilidade tem se destacado como uma das principais metodologias de criação de indicadores de sustentabilidade. Neste trabalho apresentamos uma proposta denominada 'barometroR', baseada em um algoritmo que visa a simplificação do número de atributos necessários para obtenção do índice de sustentabilidade. Para validação dos resultados, são comparados dois métodos de pré-processamento para seleção de características com o modelo original baseado na literatura, em um estudo de caso para 13 municípios, em que se avaliam 26 atributos. Os resultados demonstram que a metodologia proposta garante a mesma pontuação do Barômetro com intervalo de confiança de $95 \%$ selecionando 10 atributos.

\section{Introdução}

O desenvolvimento sustentável tem sido defendido com maior intensidade nas últimas cinco décadas a partir de diversas entidades locais, regionais, nacionais e internacionais [Beni 2003], [Nascimento 2012]. Apesar do esforço, os avanços de proteção ambiental ainda podem ser considerados tímidos, quando comparados ao aumento anual de áreas desmatadas e queimadas causadas pelo homem e seu capital.

Para solucionar essa problemática, diversas agendas defendem o constante monitoramento de áreas em risco e a implantação de políticas públicas que respondam às demandas criadas por inúmeras dinâmicas sociais, ambientais e econômicas [Ministério do Meio Ambiente 2021], [Nações Unidas do Brasil 2021] [Nova Agenda Urbana 2019]. Deste modo, sendo necessária a definição de um consenso metodológico que possa definir parâmetros para uma adequada implantação de uma gestão que respeite a sustentabilidade e os interesses da população estabelecida na localidade. Para esse modelo de gestão, se faz necessário levar em consideração metodologias que considerem de forma sistemática vários parâmetros, tais como, saúde, economia, 
educação, qualidade do ar, condições de habitação, entre outros. Neste contexto, o Barômetro da Sustentabilidade (BS) foi proposto por Robert Prescott-Allen em 1942 com o intuito de servir como ferramenta capaz de sumarizar diferentes características a respeito do bem-estar humano e o bem-estar ecológico presentes em diferentes municípios e regiões do mundo [Silva e Vieira 2016], [Cardoso, de Toledo e Vieira 2016]. Destacando-se como um instrumento replicável em diferentes cenários.

Embora o BS possua as vantagens destacadas, seu maior desafio para sua aplicação prática diz respeito à necessidade da coleta de informações, que muitas das vezes não estão disponíveis de forma fácil, adequada e rápida para a população ou órgãos competentes e identificação de variáveis relevantes [de Oliveira et al. 2015]. Diante deste contexto desafiador, este trabalho apresenta uma proposta de solução baseada em um algoritmo para classificação dos índices de sustentabilidade a partir da linguagem de programação $\mathrm{R}$ considerando um número menor de dados a serem coletados. Para isso, é proposto uma metodologia de seleção e pré-processamento dos dados com o intuito de podar o número de variáveis a serem coletadas, de modo a manter a qualidade e confiabilidade dos resultados.

O restante deste trabalho está organizado da seguinte forma. Na seção 2 é apresentada a teoria em relação ao BS. Já na seção 3 é apresentada a metodologia proposta neste trabalho. Nas seções 4 e 5 são apresentados, respectivamente, os resultados e considerações finais do trabalho. Por fim, a seção 6 apresenta as referências adotadas nesta investigação.

\section{Barômetro da Sustentabilidade}

De forma conceitual, o BS é uma metodologia utilizada para medir o nível de bem-estar levando em consideração dois sistemas, sendo eles, humano e ambiental. Em sua metodologia, o BS propõe a aplicação de critérios de desempenho, objetivos e indicadores para classificação em cinco níveis de sustentabilidade que contemplem as questões centrais para medição do desenvolvimento sustentável e o bem-estar da população.

O BS evolui como um framework, no qual podem ser customizadas as dimensões, temas, variáveis e a métrica de pontuação, o que permite a adequação da metodologia a partir dos conhecimentos de pesquisadores especialistas ou, como neste trabalho, a partir da aplicação de algoritmos computacionais. Deste modo, o BS pode fornecer flexibilidade para que estudos abrangentes possam lidar com informações ausentes ou dissonantes da realidade.

O pioneirismo da aplicação do BS no Brasil foi dado por Kronemberger (2003), sendo este acompanhado por outros autores e suas produções que aplicaram a metodologia em diferentes contextos e dimensões territoriais do Brasil [Kronemberger et. al 2008], [Cetrulo, Molina e Medeiros 2013] . Além dos trabalhos acadêmicos, o BS 
também passou a ser tema de interesse de órgãos públicos, visto que a metodologia teve boa adequação na tarefa de monitoramento, controle e fiscalização das ações, metas e objetivos de governos [Nações Unidas no Brasil 2021].

A modelagem matemática do BS foi apresentada por [Kronemberger, Carvalho e Junior 2004], sendo esta baseada na construção de escalas de desempenho. Os valores da Escala de Desempenho Municipal (EDM) são adotados no cálculo das Escalas do Barômetro da Sustentabilidade (EBS), conforme demonstrado na equação (1):

$$
B S_{X}=\left\{\left[\frac{\left(D M_{A}-D M_{x}\right) \times\left(B S_{A}-B S_{P}\right)}{\left(D M_{A}-D M_{P}\right)}\right] \times(-1)\right\}+B S_{A}
$$

onde DM denota o desempenho municipal para o indicador manipulado; $A$ e $P$ indicam, respectivamente, o limite anterior do intervalo que contém $X$ e o limite posterior do intervalo que contém $X$.

\section{Metodologia}

Neste trabalho a metodologia foi dividida em 3 Etapas, sendo elas extração dos dados; seleção/pré-processamento e cálculo; e análise dos dados. A Figura 1 ilustra a estrutura da organização do algoritmo a partir de suas etapas para a obtenção dos resultados.

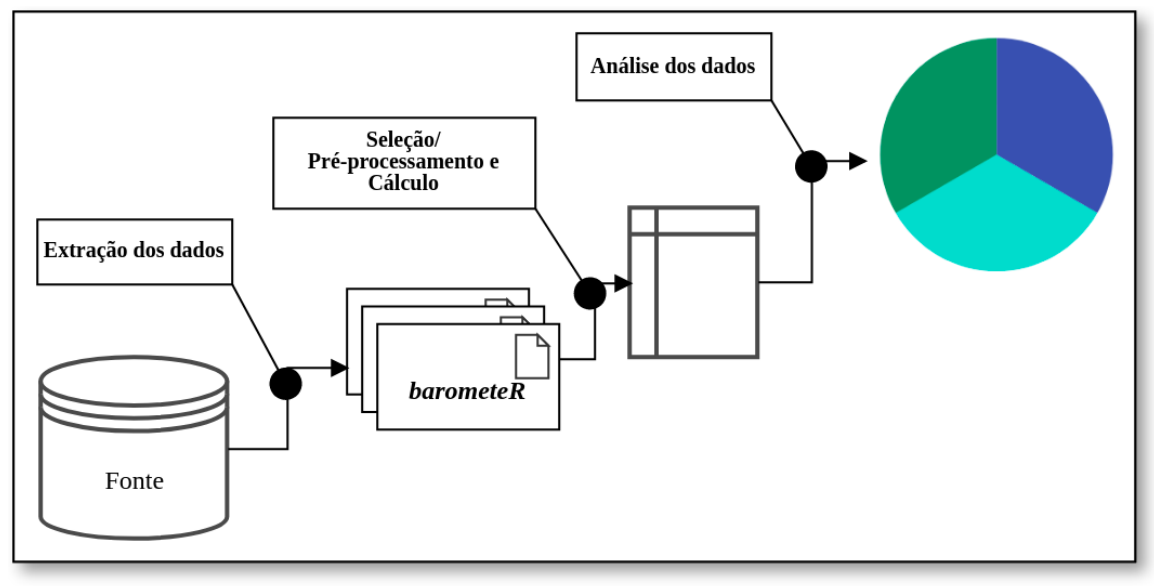

Figura 1: Ilustração do fluxograma da metodologia proposta.

Na Etapa 1 foram coletados os dados de 13 municípios do estado do Pará, a partir dos relatórios disponibilizados pela FAPESPA ${ }^{1}$ [FAPESPA 2019]. Durante a coleta, os dados foram filtrados e organizados em tabela do tipo csv (comma-separated values), tendo as linhas como variáveis ${ }^{2}$ e municípios como colunas. Na Etapa 2 foram realizadas duas tarefas principais. A primeira tarefa foi a implementação em linguagem

\footnotetext{
1 A Fundação Amazônia de Amparo a Estudos e Pesquisas do Pará (FAPESPA) é um órgão governamental que gerencia os recursos de pesquisa, desenvolvimento e sustentabilidade do Governo do Estado.

${ }^{2}$ A variável ou atributo corresponde a uma característica que pode ser representado por um valor numérico, literal ou booleano, por exemplo, a renda per capita no município sob investigação.
} 
$\mathrm{R}$ do modelo matemático do BS proposto por Prescott-Allen (2001) e adotado pela FAPESPA. Com a codificação, os resultados apresentados nos documentos do Governo foram reproduzidos para futura comparação com os resultados do método proposto no trabalho. Já a segunda tarefa consistiu na implementação da solução denominada 'barometroR'. O 'barometroR' é uma solução proposta que conta com a implementação de um algoritmo que pode ser dividido em três passos, sendo eles, seleção de dados, pré-processamento dos dados e cálculo do barômetro.

O passo de seleção e pré-processamento consiste na etapa de aplicação do método de seleção de características comuns entre os atributos. Este passo visa reduzir a quantidade de variáveis a serem processadas durante o cálculo do BS, assim selecionando estatisticamente os atributos que mais contribuem para a explicação do fenômeno. Para a realização deste passo são propostos dois métodos, sendo o primeiro baseado em correlação e o segundo baseado em Principal Component Analysis (PCA), sendo em seguida comparados com o método original na etapa de análise.

O resultado da correlação é dado por uma matriz quadrada que pode ser interpretada a partir da comparação entre covariância de atributos, isto é, quanto o atributo $A$ varia na mesma medida que B também varia. Esses valores são normalizados de 1 a -1 , sendo valores mais próximos à 1 uma indicação de covariação positiva forte; enquanto que valores próximos à zero indicam uma correlação fraca; por fim, valores mais próximos à -1 indicam uma variação igualmente forte e inversa. Após o cálculo da correlação, foi realizado o ranqueamento dos resultados em função das variáveis que apresentaram maior valor absoluto de coeficiente de correlação em relação a média das outras variáveis, conforme formalizado na equação (2). Sabendo que todas os atributos submetidos à análise são interdependentes, podemos então representar os resultados como sendo a matriz $\mathrm{A}_{\mathrm{ij}}$ com os valores correspondentes à associação do conjunto de municípios $\mathrm{i}=\left\{\mathrm{i}_{o}, \mathrm{i}_{1}, \mathrm{i}_{2}, \ldots \mathrm{i}_{k}\right\}$, para um $k=13$ e $\mathrm{j}^{D}=\left\{\mathrm{j}_{o}, \mathrm{j}_{1}, \mathrm{j}_{2}, \ldots \mathrm{j}_{p}\right\}$ representando o conjunto de atributos $A$ na dimensão $D$, onde $p$ e $D$ representam respectivamente, o número de atributos na dimensão $D$ e a dimensão $D$ categorizada em bem-estar Humano ou Ambiental.

$$
F_{\text {corr }}^{D}=\operatorname{rank}\left[\operatorname{abs}\left(\operatorname{corr}\left(A_{i j}\right)_{i p}\right)\right]
$$

A análise de componentes principais é uma técnica de análise multivariada que consiste em transformar as coordenadas de uma maneira orientada ao eixo de maior variação dos dados à medida que preserva a ortogonalidade, e os dados são representados em função da ordem decrescente da máxima variância (componentes), permitindo observar a contribuição de cada variável para a representação dos dados [Neto e Moita 1998]. Para reduzir o efeito da escala de cada variável os dados foram normalizados. Em seguida, os atributos foram ranqueados considerando as suas contribuições relativas na explicação da primeira componente do PCA. Ao final, da etapa de pré-processamento é retornada uma matriz reduzida, que por sua vez, é 
composta por todos os municípios/registros e pelo número reduzido de atributos a serem apresentados ao BS. Por fim, a matriz reduzida é utilizada para o cálculo do BS, de onde são obtidas as pontuações de cada variável, podendo ser agrupadas pela média em temas ou dimensões.

Na Etapa 3 os dados originais e calculados a partir do algoritmo proposto neste trabalho são submetidos ao teste de normalidade de Shapiro-Wilks e comparados utilizando o teste $t$ pareado com um nível de significância de 5\%, adotando-se como hipótese nula que não há diferenças significativas entre as pontuações. Os resultados das pontuações que atendem aos requisitos do teste são convertidas para os níveis de sustentabilidade do barômetro e podem ser impressos graficamente para análise e comparação. A comparação permite verificar a classificação de cada município em relação aos índices de sustentabilidade, sendo o objetivo de que o resultado do algoritmo, mesmo com um número menor de atributos, seja o mesmo indicado pela forma original de medição da sustentabilidade.

\section{Estudo de Caso}

Como pontua [de Souza Amorim, Araújo e Cândido 2014] a escolha de indicadores normalmente está associada à existência, consistência dos dados e facilidade de mensuração, nesse sentido é válido reforçar que este estudo de caso faz uso de dados obtidos a partir dos relatórios oficiais acessíveis pelo sítio eletrônico da FAPESPA [FAPESPA 2019]. Como resultado do BS serão retornados valores em escala determinados entre o intervalo de 0 até 100 , onde os valores abaixo de 20 serão considerados insustentáveis; abaixo de 40 serão determinados como potencialmente insustentáveis; abaixo de 60 serão classificados como intermediários; acima de 60 serão indicados como potencialmente sustentáveis; por fim, os valores acima de 80 serão classificados como sustentáveis. Neste trabalho a escala é denominada de EDM, tendo seus limites estabelecidos pelo especialista do domínio.

Sobre o algoritmo proposto, este teve como dados de entrada os valores de 26 atributos relacionados aos cinco temas associados ao padrão de bem-estar humano e quatro temas associados ao bem-estar ambiental. A Tabela 1 sumariza os atributos em relação a sua dimensão, tema, fonte de dados e unidade numérica dos dados.

Neste estudo de caso, também foi levado em consideração o comportamento de interesse dos atributos de entrada, isto é, existem variáveis que possuem evoluções inversas à EBS, por exemplo, a variável taxa de mortalidade infantil tem uma curva decrescente em relação à EBS, uma vez que quanto menor a variável, mais se avança para o desenvolvimento sustentável; enquanto que a variável número de médicos tem tendência crescente já que quanto maior a variável, maior o nível de desenvolvimento sustentável, essa tendência de crescimento também deve ser indicada pelo pesquisador. 
Para avaliar a eficiência do algoritmo no cálculo do BS e a validade da hipótese levantada, criamos seis cenários experimentais agrupados pela técnica de análise fatorial aplicada durante o pré-processamento (Correlação e PCA) e quantidade de variáveis explicativas (3, 4 e 5), além do cenário original com todas as variáveis. Considerando uma significância de 5\%, todas as variáveis foram submetidas à teste de normalidade de Shapiro-Wilk para garantir os pressupostos estatísticos para o teste $t$ pareado, no qual são confrontados os valores do BS correspondentes ao banco de dados original e suas formas reduzidas, adotando-se como hipótese nula que não há diferenças entre as médias.

\begin{tabular}{|c|c|c|c|}
\hline \multicolumn{4}{|c|}{ Tabela 1: Estrutura do Barômetro da Sustentabilidade adotado pela FAPESPA. } \\
\hline TEMA & FONTE DE DADOS ${ }^{3}$ & INDICADOR & UNIDADE \\
\hline \multirow{6}{*}{$(1)^{*}$} & \multirow{5}{*}{ DATASUS 2018} & Mortalidade na infância & Mortes/1000 habitantes \\
\hline & & Mortalidade materna & Mortes/1.000 habitantes \\
\hline & & Número de médicos & Médicos/1000 habitantes \\
\hline & & Leitos hospitalares & Leitos/1.000 habitantes \\
\hline & & Gravidez na infância e adolescência & $\%$ \\
\hline & \multirow{6}{*}{$\begin{array}{l}\text { IBGE - Censo } \\
\text { Demográfico } 2010\end{array}$} & Extrema pobreza & $\%$ \\
\hline \multirow{5}{*}{$(2)^{*}$} & & Taxa de atividade & $\%$ \\
\hline & & Trabalho infantil & $\%$ \\
\hline & & PIB (per capita) & Valor (R\$ mil) \\
\hline & & Renda (per capita) & Valor (R\$ mil) \\
\hline & & Analfabetismo & $\%$ \\
\hline \multirow{6}{*}{$(3)^{*}$} & \multirow{4}{*}{ INEP 2017} & Ideb (séries iniciais) & Nota \\
\hline & & Ideb (séries finais) & Nota \\
\hline & & \multicolumn{2}{|l|}{ Evasão escolar no Ensino Fundamental \% } \\
\hline & & Evasão escolar no Ensino Médio & $\%$ \\
\hline & \multirow{2}{*}{$\begin{array}{l}\text { IBGE - Censo } \\
\text { Demográfico } 2010 \\
\text { FAPESPA } 2018\end{array}$} & Acesso à internet & \% da população \\
\hline & & Roubos & Roubos/100 mil habitantes \\
\hline \multirow[t]{2}{*}{$(4)^{*}$} & DATASUS 2018 & Homicídios & $\begin{array}{l}\text { Homicídios/100 mil } \\
\text { habitantes }\end{array}$ \\
\hline & $\begin{array}{l}\text { IBGE - Censo } \\
\text { Demográfico } 2010\end{array}$ & $\begin{array}{l}\text { Acesso à energia elétrica } \\
\text { Índice de Gini }\end{array}$ & $\begin{array}{l}\% \text { da população em } \\
\text { domicílios } \\
0 \text { a } 1\end{array}$ \\
\hline \multirow{2}{*}{$(6)^{*}$} & SEMAS 2018 & Cadastro ambiental rural & $\%$ \\
\hline & PRODES 2018 & Desmatamento & $\mathrm{km}^{2} / \mathrm{ano}$ \\
\hline \multirow{2}{*}{$(7)^{*}$} & IBGE - Censo & $\begin{array}{l}\text { População em domicílios com água } \\
\text { encanada }\end{array}$ & $\begin{array}{l}\text { \% da população em } \\
\text { domicílios }\end{array}$ \\
\hline & $\begin{array}{l}\text { IBGE - Censo } \\
\text { Demográfico } 2010\end{array}$ & \multicolumn{2}{|c|}{ População em domicílios com banheiro \% da população em } \\
\hline$(8)^{*}$ & Inpe Queimadas 2018 & Focos de calor & Focos $/ 1.000 \mathrm{~km}^{2} / \mathrm{ano}$ \\
\hline$(9)^{*}$ & $\begin{array}{l}\text { IBGE - Censo } \\
\text { Demográfico } 2010\end{array}$ & Coleta de lixo & $\begin{array}{l}\text { \% da população em } \\
\text { domicílios }\end{array}$ \\
\hline $\begin{array}{r}\text { *Legenda } \\
\text { Bem-e } \\
(1) \\
\text { Ambier } \\
(6) \\
\text { Fonte: Ad }\end{array}$ & $\begin{array}{l}\text { star humano: } \\
\text { Saúde e População; (2) Riqueza } \\
\text { ntal: } \\
\text { Terra; (7) Água; (8) Ar; e (9) Us } \\
\text { laptado do Relatório do barômet }\end{array}$ & $\begin{array}{l}\text {; (3) Conhecimento e cultura; (4) Comunidade; e (5 } \\
\text { so de recursos naturais. } \\
\text { tro da Sustentabilidade do município de Castanhal// }\end{array}$ & (5) Equidade. \\
\hline
\end{tabular}

\section{Resultados}

\footnotetext{
${ }^{3}$ As fontes primárias foram consultadas pela FAPESPA (2019).
} 
É válido reforçar que o BS é uma metodologia eficiente devido a sua aplicação direta e alta flexibilidade na aquisição dos dados, interpretação e análise dos resultados. A fonte de dados utilizada neste trabalho apresenta os resultados em dois níveis hierárquicos (dimensão e tema). No entanto, o nível temático tem menor potencial de comparação, já que usualmente o BS é apresentado de forma bi-dimensional (eixo de bem-estar humano e eixo de bem-estar ambiental). A Tabela 2, detalha os valores do barômetro da sustentabilidade em nível temático para os 13 municípios estudados.

\begin{tabular}{|c|c|c|c|c|c|c|c|}
\hline \multirow[b]{3}{*}{ MUNICÍPIOS } & \multicolumn{7}{|c|}{ Tabela 2: Valores do Barômetro da Sustentabilidade por temática } \\
\hline & & & & TEMA & & & \\
\hline & $\mathrm{Ar}$ & Comunidade & $\begin{array}{c}\text { Conhecimento } \\
\text { e cultura }\end{array}$ & Equidade & Riqueza & $\begin{array}{c}\text { Saúde e } \\
\text { população }\end{array}$ & Terra \\
\hline $\begin{array}{l}\text { Bom Jesus do } \\
\text { Tocantins }\end{array}$ & 63.78 & 44.96 & 37.79 & 38.67 & 41.62 & 51.54 & 88.76 \\
\hline Brejo Grande & 79.82 & 50.04 & 37.10 & 35.33 & 40.64 & 64.87 & 91.08 \\
\hline $\begin{array}{l}\text { Canaã dos } \\
\text { Carajás }\end{array}$ & 88.56 & 41.08 & 49.74 & 36.67 & 59.62 & 57.63 & 91.00 \\
\hline Castanhal & 55.30 & 42.66 & 48.69 & 37.33 & 57.32 & 60.17 & 78.73 \\
\hline Curionópolis & 75.52 & 43.61 & 43.79 & 37.33 & 55.21 & 61.85 & 95.61 \\
\hline $\begin{array}{l}\text { Eldorado dos } \\
\text { Carajás }\end{array}$ & 62.12 & 32.93 & 38.42 & 35.33 & 37.44 & 51.60 & 97.22 \\
\hline Marabá & 53.20 & 40.17 & 46.50 & 34.67 & 56.05 & 35.75 & 81.76 \\
\hline Parauapebas & 87.52 & 43.98 & 56.79 & 38.00 & 66.00 & 36.36 & 89.59 \\
\hline Piçarra & 75.24 & 51.38 & 38.16 & 38.67 & 42.92 & 50.25 & 90.59 \\
\hline $\begin{array}{l}\text { São Domingos } \\
\text { do Araguaia }\end{array}$ & 78.46 & 44.81 & 40.51 & 34.00 & 42.32 & 49.42 & 92.34 \\
\hline $\begin{array}{l}\text { São Geraldo } \\
\text { do Araguaia }\end{array}$ & 84.86 & 57.70 & 41.53 & 38.00 & 46.33 & 57.57 & 93.90 \\
\hline $\begin{array}{l}\text { São João do } \\
\text { Araguaia }\end{array}$ & 25.00 & 44.29 & 31.69 & 36.67 & 33.74 & 56.03 & 86.90 \\
\hline $\begin{array}{l}\text { Palestina do } \\
\text { Pará }\end{array}$ & 69.52 & 54.95 & 38.11 & 36.67 & 38.07 & 66.10 & 94.49 \\
\hline
\end{tabular}

Na temática qualidade do ar, o município de São João do Araguaia apresentou um nível potencialmente insustentável, em função da quantidade de focos de calor registrados na área do município.

Analisando graficamente a distribuição do BS dimensional na figura 2 percebemos que os municípios estudados estão em nível potencialmente insustentável à intermediário em relação a dimensão de bem-estar humano, assim como, em níveis intermediário à potencialmente sustentável no que diz respeito à dimensão de bem-estar ambiental.

Ao considerar o efeito da seleção de características sobre o BS foi observado que existe um número mínimo de atributos para manter os resultados consistentes, não sendo recomendável selecionar menos de 5 atributos por dimensão. Os atributos mais relevantes contribuem para que as diferenças absolutas não sejam estatisticamente significativas, desse modo, garantindo que o algoritmo proposto retorne pontuações equivalentes ao Barômetro da Sustentabilidade original, isto é, dentro do intervalo de confiança considerado, mesmo utilizando um menor número de atributos. A partir do 
limite mínimo identificado pelo método, os resultados mais precisos podem ser obtidos considerando um número maior de atributos, conforme necessidades da pesquisa.

A Figura 2 ilustra o resultado obtido pelo algoritmo e compara com o obtido usando os dados originais. A partir dos resultados, é possível verificar que o algoritmo proposto classificou corretamente $61,54 \%$ dos municípios pela estratégia que fez uso do método de seleção de característica baseada em correlação; e 73,08\% com o PCA, assim como, conseguiu reduzir o número de atributos para classificação de 26 para 10, sendo eles 5 da dimensão Humana e 5 da dimensão ambiental. A precisão na dimensão humana foi de $69,23 \%$ para ambos os métodos aplicados no pré-processamento (baseado em correlação e PCA); por outro lado, a precisão da dimensão ambiental foi de $53,85 \%$ com o método baseado em correlação, e de 76,92\% pelo método de PCA. A diferença existente na dimensão ambiental é explicada pelo menor número de variáveis no conjunto original (apenas 6 variáveis) resultando num impacto muito maior da variância na substituição ou remoção de uma única variável, enquanto que a dimensão humana parte de 21 variáveis sendo menos afetada pela etapa de pré-processamento.

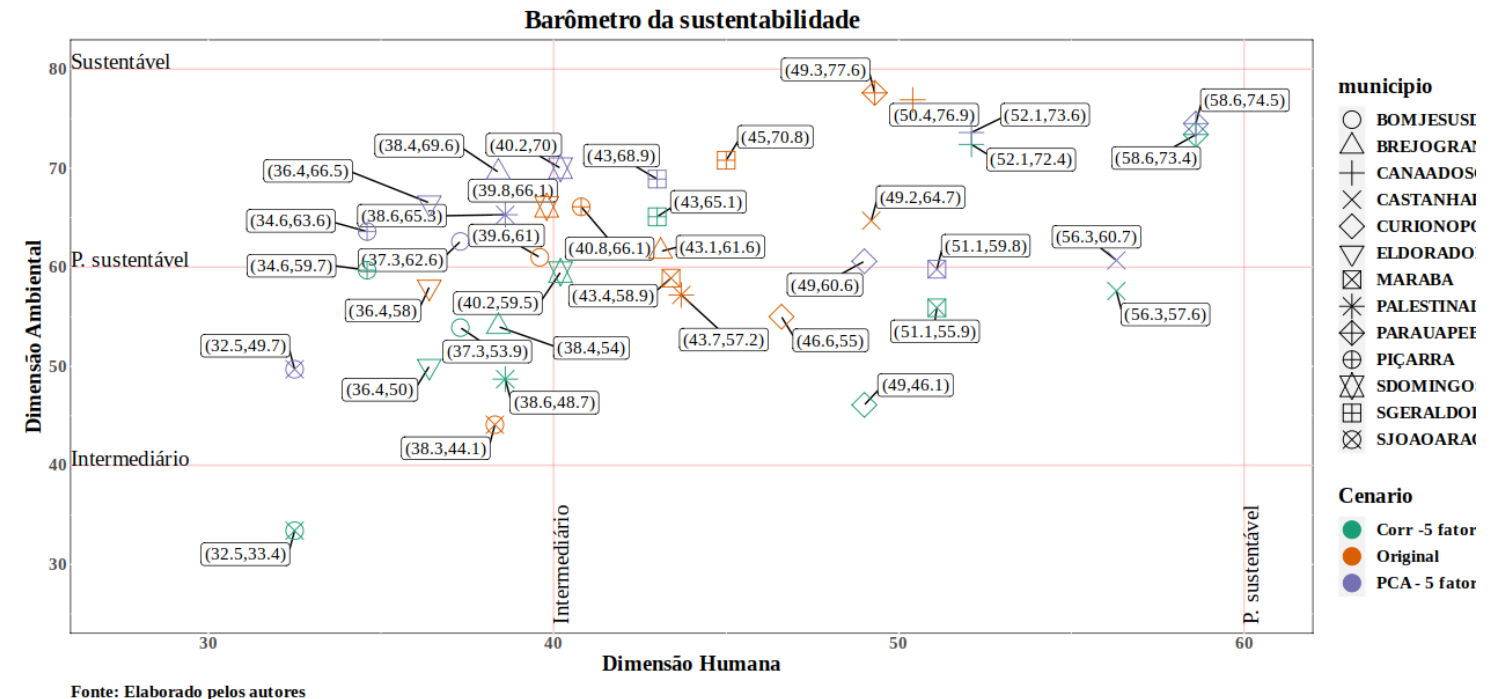

Figura 2. Gŕafico com representação dimensional do Barômetro da Sustentabilidade para o banco de dados reduzido comparado ao banco de dados completo.

Os resultados do método de pré-processamento com o método de seleção de características baseado em correlação indicam que os cinco atributos a serem considerados na dimensão ambiental são: População em domicílios com banheiro e água encanada, Cadastro Ambiental Rural, População em domicílios com água encanada, Desmatamento e Coleta de lixo. Já para a dimensão humana foram selecionados os atributos: Acesso à Internet, PIB per capita, Trabalho infantil, Taxa de atividade e Extrema pobreza . Em relação ao método de pré-processamento com PCA a dimensão ambiental foi melhor representada por: Cadastro Ambiental Rural, População em domicílios com água encanada, População em domicílios com banheiro, Focos de 
calor e Desmatamento; enquanto a dimensão humana foi melhor representada pelas variáveis de Acesso à Internet, Trabalho infantil, Taxa de atividade, Extrema pobreza e Acesso à energia elétrica.

\section{Considerações Finais e Trabalhos Futuros}

Este trabalho apresentou um novo algoritmo para definição do índice de sustentabilidade de municípios. O algoritmo proposto é dividido nas etapas de seleção, pré-processamento e análise, sendo adotado na etapa de pré-processamento os métodos estatísticos baseados em correlação e PCA para filtragem de atributos. Ao final, os resultados do BS original, e do algoritmo com processamento de correlação e PCA foram comparados. A partir dos resultados é possível concluir que o algoritmo proposto resultou em um resultado estatisticamente condizente com a amostra analisada para um intervalo de confiança de $95 \%$ para o cálculo do BS, no entanto, para a sua classificação em nível de sustentabilidade houveram divergências em relação ao conjunto de dados original, sobretudo em função da quantidade de registros implicando em uma maior variância.

Os resultados apresentados no trabalho demonstram que o trabalho de aquisição de dados pode ser minimizado a partir de uma boa razão entre quantidade e qualidade das variáveis, já que muitas variáveis resultam em um maior trabalho de coleta e tratamento. Também elencamos algumas variáveis com maior poder explicativo no estudo de caso, e disponibilizamos uma ferramenta para aplicação da metodologia em outros cenários.

A partir deste trabalho é possível concluir que algoritmos de propósito específico implementados em linguagem $\mathrm{R}$ podem ser poderosas ferramentas para otimizar $\mathrm{o}$ processo de identificação do nível de sustentabilidade de municípios mesmo com a redução do número de atributos necessários para classificação. Como trabalhos futuros, espera-se realizar testes com dados de outras regiões do Brasil, assim como, expandir a ferramenta para uso web incorporando outras técnicas de seleção de atributos.

\section{Referências}

Beni, M. C. (2003). Como certificar o turismo sustentável?. Revista Turismo em Análise, 14(2), 5-16.

Cardoso, A. S., de Toledo, P. M. e Vieira, I. C. G. (2016). Barômetro da sustentabilidade aplicado ao município de Moju, estado do Pará. Revista Brasileira de Gestão e Desenvolvimento Regional, 12(1).

Cetrulo, T. B., Molina, N. S. e Malheiros, T. F. (2013). Indicadores de sustentabilidade: proposta de um barômetro de sustentabilidade estadual. Brazilian Journal of Environmental Sciences (Online), (30), 33-45. 
Ministério do Meio Ambiente. Caderno de Debates - Agenda 21 e Biodiversidade. Governo Federal. Disponível em: $<$ https://antigo.mma.gov.br/estruturas/agenda21/ arquivos/CadernodeDebates9.pdf>. Acessado em: 27/05/2021.

de Oliveira, E. L., Oliveira, E. A. D. A. Q., \& Carniello, M. F. (2015). O Barômetro da Sustentabilidade aplicado ao município de Taubaté-SP. Desenvolvimento em Questão, 13(30), 230-264.

de Souza Amorim, A., Araújo, M. F. F. e Cândido, G. A. (2014). Uso do barômetro da sustentabilidade para avaliação de um município localizado em região Semiárida do Nordeste Brasileiro. Desenvolvimento em questão, 12(25), 189-217.

FAPESPA (Fundação Amazônia de Ampara a Estudos e Pesquisas), 2019. Barômetro da Sustentabilidade 2019. Governo do Pará, Belém. Disponível em: <http://www.fapespa.pa.gov.br/upload/Arquivo/anexo/2256.pdf?id=1614820479>. Acessado em: 06/03/2020

Kronemberger, D.M.P. (2003) A Viabilidade do Desenvolvimento Sustentável na Escala Local: o caso da Bacia do Jurumirim (Angra dos Reis, RJ). Tese de Doutoramento. Instituto de Química, Universidade Federal Fluminense, 274 p.

Kronemberger, D. M. P., Junior, J. C., Nascimento, J. A. S., Collares, J. E. R. e Silva, L. C. D. (2008). Desenvolvimento sustentável no brasil: uma análise a partir da aplicação do barômetro da sustentabilidade. Sociedade \& Natureza, 20(1), 25-50.

Kronemberger, D. M. P., Carvalho, C. N. e Junior, J. C. (2004). Indicadores de sustentabilidade em pequenas bacias hidrográficas: uma aplicação do "Barômetro da Sustentabilidade" à bacia do Jurumirim (Angra dos Reis/RJ). Geochimica Brasiliensis, 18(2).

Neto, J. M. M. e Moita, G. C. (1998). Uma introdução à análise exploratória de dados multivariados. Química nova, 21(4), 467-469.

Nascimento, E. P. D. (2012). Trajetória da sustentabilidade: do ambiental ao social, do social ao econômico. Estudos avançados, 26(74), 51-64.

Nações Unidas no Brasil. Disponível em <https://brasil.un.org/pt-br/sdgs $>$. Acesso em $15 / 04 / 2021$.

Prescott-Allen, R. (2001). The wellbeing of nations: A country-by-country Index of Quality of Life and the Environment. Island Press.

Silva, V. C. S. e Vieira, I. C. G. (2016). Barômetro da Sustentabilidade aplicado a assentamentos rurais do leste do Estado do Pará, Brasil. Desenvolvimento e Meio Ambiente, 36. 